MATHEMATICS OF COMPUTATION

Volume 72, Number 244, Pages 1635-1653

S 0025-5718(03)01508-4

Article electronically published on March 4, 2003

\title{
ANALYSIS OF A BILINEAR FINITE ELEMENT FOR SHALLOW SHELLS. II: CONSISTENCY ERROR
}

\author{
VILLE HAVU AND JUHANI PITKÄRANTA
}

\begin{abstract}
We consider a bilinear reduced-strain finite element of the MITC family for a shallow Reissner-Naghdi type shell. We estimate the consistency error of the element in both membrane- and bending-dominated states of deformation. We prove that in the membrane-dominated case, under severe assumptions on the domain, the finite element mesh and the regularity of the solution, an error bound $O\left(h+t^{-1} h^{1+s}\right)$ can be obtained if the contribution of transverse shear is neglected. Here $t$ is the thickness of the shell, $h$ the mesh spacing, and $s$ a smoothness parameter. In the bending-dominated case, the uniformly optimal bound $O(h)$ is achievable but requires that membrane and transverse shear strains are of order $O\left(t^{2}\right)$ as $t \rightarrow 0$. In this case we also show that under sufficient regularity assumptions the asymptotic consistency error has the bound $O(h)$.
\end{abstract}

\section{INTRODUCTION}

Approximation of deformation states arising in thin shells by low-order finite element methods is known to be a nontrivial task. Different locking modes degrade the convergence rate of the most basic formulations when approximating bendingdominated or inextensional deformations. However, it is equally well-known by now that a suitable variational crime can be used to retain the convergence properties in such cases. This can even be done up to an optimal order and smoothness requirements for certain shell geometries, as was shown in Part I of this paper [3], see also [7]. The real challenge begins when one aims to find a formulation that has a satisfactory behavior also in the membrane-dominated states of deformation. In this case one is inevitably led to consider the questions of consistency and stability of the formulation, since the approximation properties will rarely be a problem in such a case, but lack of consistency or stability can yield a very large error component.

Probably most low-order shell elements that aim to be general in nature contain the basic ideas of MITC4 by Bathe and Dvorkin [1. In [4] it was shown that this formulation is in fact equivalent to a certain variational crime already considered in [7. In this paper we extend our analysis of the MITC-type elements and address their consistency and stability properties. We show that, at least under favorable circumstances, this kind of an element can indeed also approximate membranedominated deformation well. However, due to the lack of stability in the membranedominated case, we can bound the consistency error in this case only non-uniformly

Received by the editor January 18, 2001 and, in revised form, February 7, 2002.

2000 Mathematics Subject Classification. Primary 65N30; Secondary 73K15.

Key words and phrases. Finite elements, locking, shells. 
with respect to the thickness $t$ of the shell. We also need strong assumptions on the problem setup and on the finite element mesh, as in the previous part [3]. Under such hypotheses and under certain additional hypotheses on the solution, we show that the consistency error is at most of order $O\left(h+t^{-1} h^{1+s}\right)$, where $h$ is the mesh spacing and $s \geq 0$ is a parameter depending on the degree of smoothness of the exact solution. As $s$ can be arbitrarily large in principle, one can have $t^{-1} h^{s}=O(1)$ for reasonable sequences of $(t, h)$ if the solution is very smooth. In such a case the consistency error is $O(h)$, which is the optimal order for bilinear elements.

Another topic to be considered in this paper is the asymptotic behavior of the consistency error in the case of an inextensional deformation. In 3 we considered the problem of finding a best finite element approximation of a given inextensional deformation. At that point the question of consistency was deliberately left aside. However, in real computations one is inevitably faced with the fact that since the reduced inextensional space is not a subspace of the corresponding continuous space, the consistency error does not tend to zero when the thickness $t \rightarrow 0$, but to some finite value depending on $h$. Here we show that this error term is of the optimal order $O(h)$. However, to obtain this result we need much stronger regularity assumptions on the exact solution than in the previous Part I [3], where we bounded the approximation error. Whether our analysis here is sharp is not clear at the moment.

The plan of this paper is as follows. In section 2 we describe the problems to be considered and in section 3 we consider two slightly different FEM approximations to these. Section 4 is devoted to the consistency error in the non-asymptotic case $(t>0)$, whereas section 5 deals with the asymptotic consistency error in the inextensional deformation state. In section [6 we draw the conclusions of Parts I and II of the paper.

In the following we denote by $C$ a generic constant that may take a different value each time. The constants may depend on the geometry parameters of the problem but are otherwise independent of the parameters, unless indicated explicitly. The Sobolev norm and seminorm are denoted by $\|\cdot\|_{k}$ and $|\cdot|_{k}$ respectively on the assumed rectangular domain. Further, $\|\cdot\|_{L^{2}}=\|\cdot\|_{0}$, and $(\cdot, \cdot)$ denotes the $L^{2}$ inner product.

\section{THE SHELL PROBLEM}

We use basically the same shell model of Reissner-Naghdi type as in 3], but with two different scalings. Denoting by $\underline{u}=(u, v, w, \theta, \psi)$ the vector of three translations and two rotations, we let the (scaled) total energy of the shell be given either by

$$
\mathcal{F}_{M}(\underline{u})=\frac{1}{2}\left(t^{2} \mathcal{A}_{b}(\underline{u}, \underline{u})+\mathcal{A}_{m}(\underline{u}, \underline{u})\right)-Q(\underline{u})
$$

or by

$$
\mathcal{F}_{B}(\underline{u})=\frac{1}{2}\left(\mathcal{A}_{b}(\underline{u}, \underline{u})+t^{-2} \mathcal{A}_{m}(\underline{u}, \underline{u})\right)-Q(\underline{u}),
$$

where $t$ is the thickness of the shell and $Q$ represents the load potential. Here the subscripts $M$ and $B$ refer to the natural scalings of the total energy in membraneand bending-dominated deformations, respectively. We assume that in both cases $Q(\underline{u})$ defines a bounded linear functional on the corresponding energy space to be defined later. The bilinear forms $\mathcal{A}_{b}(\underline{u}, \underline{v})$ and $\mathcal{A}_{m}(\underline{u}, \underline{v})$ arising from the bending 
and membrane energies are given by

$$
\mathcal{A}_{b}(\underline{u}, \underline{v})=\int_{\Omega}\left\{\nu\left(\bar{\kappa}_{11}+\bar{\kappa}_{22}\right)(\underline{u})\left(\kappa_{11}+\kappa_{22}\right)(\underline{v})+(1-\nu) \sum_{i, j=1}^{2} \bar{\kappa}_{i j}(\underline{u}) \kappa_{i j}(\underline{v})\right\} d x d y
$$

and

$$
\begin{array}{r}
\mathcal{A}_{m}(\underline{u}, \underline{v})=6 \gamma(1-\nu) \int_{\Omega}\left\{\bar{\rho}_{1}(\underline{u}) \rho_{1}(\underline{v})+\bar{\rho}_{2}(\underline{u}) \rho_{2}(\underline{v})\right\} d x d y \\
+12 \int_{\Omega}\left\{\nu\left(\bar{\beta}_{11}+\bar{\beta}_{22}\right)(\underline{u})\left(\beta_{11}+\beta_{22}\right)(\underline{v})\right. \\
\left.+(1-\nu) \sum_{i, j=1}^{2} \bar{\beta}_{i j}(\underline{u}) \beta_{i j}(\underline{v})\right\} d x d y
\end{array}
$$

where overbars denote complex conjugation. Here $\nu$ is the Poisson ratio of the material, $\gamma$ is a shear correction factor and $\kappa_{i j}, \beta_{i j}$ and $\rho_{i}$ represent the bending, membrane and transverse shear strains respectively, depending on $\underline{u}$ as

$$
\begin{aligned}
\beta_{11} & =\frac{\partial u}{\partial x}+a w, & \kappa_{11} & =\frac{\partial \theta}{\partial x} \\
\beta_{22} & =\frac{\partial v}{\partial y}+b w, & \kappa_{22} & =\frac{\partial \psi}{\partial y} \\
\beta_{12} & =\frac{1}{2}\left(\frac{\partial u}{\partial y}+\frac{\partial v}{\partial x}\right)+c w=\beta_{21} & \kappa_{12} & =\frac{1}{2}\left(\frac{\partial \theta}{\partial y}+\frac{\partial \psi}{\partial x}\right)=\kappa_{21}
\end{aligned}
$$

and

$$
\rho_{1}=\theta-\frac{\partial w}{\partial x}, \quad \rho_{2}=\psi-\frac{\partial w}{\partial y} .
$$

The integration is taken over the midsurface $\Omega$ of the shell, which we assume to occupy the rectangular region $(0, L) \times(0, H)$ in the $x y$-coordinate space. We are considering the shell to be shallow and assume that the parameters $a, b$ and $c$ defining the geometry can be taken to be constants. We further note that if $a b-c^{2}>$ 0 the shell is elliptic, if $a b-c^{2}=0$ it is parabolic, and if $a b-c^{2}<0$ we have a hyperbolic shell.

The above two energy formulations lead naturally to two differently scaled variational formulations, the membrane $(M)$ and bending $(B)$ cases:

$(M)$ Find $\underline{u} \in \mathcal{U}_{M}$ such that

$$
\mathcal{A}_{M}(\underline{u}, \underline{v})=t^{2} \mathcal{A}_{b}(\underline{u}, \underline{v})+\mathcal{A}_{m}(\underline{u}, \underline{v})=Q(\underline{v}) \quad \forall \underline{v} \in \mathcal{U}_{M}
$$

(B) Find $\underline{u} \in \mathcal{U}_{B}$ such that

$$
\mathcal{A}_{B}(\underline{u}, \underline{v})=\mathcal{A}_{b}(\underline{u}, \underline{v})+t^{-2} \mathcal{A}_{m}(\underline{u}, \underline{v})=Q(\underline{v}) \quad \forall \underline{v} \in \mathcal{U}_{B}
$$

Here $\mathcal{U}_{M}$ and $\mathcal{U}_{B}$ are the membrane and bending energy spaces, respectively, which we take to be subspaces of $\left[H_{p}^{1}(\Omega)\right]^{5}$, where $H_{p}^{1}(\Omega)$ is the usual Sobolev space with periodic boundary conditions imposed at $y=0, H$. In $\mathcal{U}_{B}$ no constraints are imposed at $x=0, L$, whereas in $\mathcal{U}_{M}$ we assume the constraints $u=v=w=\theta=$ $\psi=0$ at $x=0, L$. In case $(B)$ we must also remove the rigid displacements from $\mathcal{U}_{B}$ so as to make (2.4) uniquely solvable. For the convenience of our error analysis, 
we make somewhat stronger assumptions than are needed for the well-posedness of (2.4): We introduce the set of pseudo-rigid displacements as

$$
\mathcal{Z}=\left\{\underline{v} \in\left[H_{p}^{1}(\Omega)\right]^{5} \mid \underline{v}=\sum_{i=1}^{5} C_{i} \underline{e}_{i}\right\},
$$

where $\underline{e}_{i}$ is the $i$ th pseudo-rigid body mode coinciding with the $i$ th Euclidean unit vector in our model, we assume that $Q(\underline{v})=0$ for every $\underline{v} \in \mathcal{Z}$, and we let $\mathcal{U}_{B}=\mathcal{Z}^{\perp}$ in $\left[H_{p}^{1}(\Omega)\right]^{5}$. Finally we denote the energy norms on $\mathcal{U}_{M}$ and on $\mathcal{U}_{B}$, respectively, by $\||\cdot|\|_{M}=\sqrt{\mathcal{A}_{M}(\cdot, \cdot)}$ and \|\|$\cdot\|\|_{B}=\sqrt{\mathcal{A}_{B}(\cdot, \cdot)}=t^{-1}\|\mid \cdot\| \|_{M}$.

Letting $t \rightarrow 0$ in (2.4), we obtain the inextensional formulation of the problem $(B)$ : Find $\underline{u}_{0} \in \mathcal{U}_{0}$ such that

$$
\mathcal{A}_{b}\left(\underline{u}_{0}, \underline{v}\right)=Q(\underline{v}) \quad \forall \underline{v} \in \mathcal{U}_{0},
$$

where $\mathcal{U}_{0}=\left\{\underline{v} \in \mathcal{U}_{B} \mid \mathcal{A}_{m}(\underline{v}, \underline{v})=0\right\} \subset \mathcal{U}_{B}$ is the space of inextensional deformations.

\section{The REDUCED-STRAIN FE SCHEME}

We consider the bilinear MITC4 finite element formulation of the problems (2.3) - (2.5). As in [3], we make strong assumptions on the finite element mesh so as to allow the use of Fourier methods in the error analysis.

Assume that $\Omega$ is divided into rectangular elements with node points $\left(x^{k}, y^{n}\right)$, $k=0, \ldots, N_{x}, n=0, \ldots, N_{y}$, and a constant mesh spacing $h_{y}$ in the $y$-direction, and that the aspect ratios of the elements satisfy $d^{-1} \leq h_{x}^{k} / h_{y} \leq d$ for some $d>0$, where $h_{x}^{k}=x^{k+1}-x^{k}$. To this mesh we associate the standard space $\mathcal{V}_{h} \subset H_{p}^{1}(\Omega)$ of continuous piecewise bilinear functions. We then define the FE spaces $\mathcal{U}_{M, h}$ and $\mathcal{U}_{B, h}$, respectively, as subspaces of $\mathcal{V}_{h}^{5}$ where the boundary or orthogonality conditions of problems $(M)$ and $(B)$ are enforced. The finite element formulation of problems (2.3) - 2.5 are then obtained by replacing $\mathcal{U}_{M}, \mathcal{U}_{B}$ by $\mathcal{U}_{M, h}, \mathcal{U}_{B, h}$ and by modifying the bilinear form $\mathcal{A}_{m}$ numerically as

$$
\begin{array}{r}
\mathcal{A}_{m}^{h}(\underline{u}, \underline{v})=6 \gamma(1-\nu) \int_{\Omega}\left\{\overline{\tilde{\rho}}_{1}(\underline{u}) \tilde{\rho}_{1}(\underline{v})+\overline{\tilde{\rho}}_{2}(\underline{u}) \tilde{\rho}_{2}(\underline{v})\right\} d x d y \\
+12 \int_{\Omega}\left\{\nu\left(\overline{\tilde{\beta}}_{11}+\overline{\tilde{\beta}}_{22}\right)(\underline{u})\left(\tilde{\beta}_{11}+\tilde{\beta}_{22}\right)(\underline{v})\right. \\
\left.+(1-\nu) \sum_{i, j=1}^{2} \overline{\tilde{\beta}}_{i j}(\underline{u}) \tilde{\beta}_{i j}(\underline{v})\right\} d x d y
\end{array}
$$

where $\tilde{\beta}_{i j}=R^{i j} \beta_{i j}, \tilde{\rho}_{i}=R^{i} \rho_{i}$ with suitable reduction operators $R^{i j}$ and $R^{i}$. As in [3], we choose these operators for $\beta_{i i}$ and $\rho_{i}$ to be

$$
\tilde{\beta}_{11}=\Pi_{h}^{x} \beta_{11}, \quad \tilde{\beta}_{22}=\Pi_{h}^{y} \beta_{22}, \quad \tilde{\rho}_{1}=\Pi_{h}^{x} \rho_{1} \quad \tilde{\rho}_{2}=\Pi_{h}^{y} \rho_{2},
$$

where $\Pi_{h}^{x}$ and $\Pi_{h}^{y}$ are orthogonal $L^{2}$-projections onto spaces $\mathcal{W}_{h}^{x}$ and $\mathcal{W}_{h}^{y}$ consisting of functions that are constant in $x$ and piecewise linear in $y$ or constant in $y$ and 
piecewise linear in $x$, respectively. For the term $\beta_{12}$ we consider two different alternatives:

(E1) $\tilde{\beta}_{12}=\Pi_{h}^{x y} \beta_{12}$,

(E2) $\tilde{\beta}_{12}=\beta_{12}+S_{12}$,

where $\Pi_{h}^{x y}=\Pi_{h}^{x} \Pi_{h}^{y}$ is the orthogonal $L^{2}$-projection onto elementwise constant functions, and, for every element $K$,

$$
S_{12 \mid K}=a \frac{\partial}{\partial y}\left(\Pi_{h}^{x} w\right)\left(x-h_{x}^{k} / 2\right)+b \frac{\partial}{\partial x}\left(\Pi_{h}^{y} w\right)\left(y-h_{y} / 2\right)+\left(\Pi_{h}^{x y} c w-c w\right)
$$

is essentially the term introduced in [4].

Remark 3.1. The formulation (E1) was assumed in [3], 7]. This is a straightforward interpretation of the MITC4 finite element formulation, but, as shown recently in [4], (E2) is actually a closer interpretation of MITC4. The two formulations are practically equivalent when approximating inextensional deformations, but may differ in other deformation states, as noted in [4]. Our error analysis here can only detect a small difference when approximating smooth membrane-dominated deformations, see Theorem 4.4 below.

The above definitions give rise to two different FE-schemes for solving (2.3) and (2.4):

$\left(M_{h}\right)$ Find $\underline{u}_{h} \in \mathcal{U}_{M, h}$ such that

$$
\mathcal{A}_{M}^{h}\left(\underline{u}_{h}, \underline{v}\right)=t^{2} \mathcal{A}_{b}\left(\underline{u}_{h}, \underline{v}\right)+\mathcal{A}_{m}^{h}\left(\underline{u}_{h}, \underline{v}\right)=Q(\underline{v}) \quad \forall \underline{v} \in \mathcal{U}_{M, h} .
$$

$\left(B_{h}\right)$ Find $\underline{u}_{h} \in \mathcal{U}_{B, h}$ such that

$$
\mathcal{A}_{B}^{h}\left(\underline{u}_{h}, \underline{v}\right)=\mathcal{A}_{b}\left(\underline{u}_{h}, \underline{v}\right)+t^{-2} \mathcal{A}_{m}^{h}\left(\underline{u}_{h}, \underline{v}\right)=Q(\underline{v}) \quad \forall \underline{v} \in \mathcal{U}_{B, h} .
$$

Upon passing to the limit $t \rightarrow 0$ in (3.3) we obtain a finite element formulation of the asymptotic problem (2.5): Find $\underline{u}_{h} \in \mathcal{U}_{0, h}$ such that

$$
\mathcal{A}_{b}\left(\underline{u}_{h}, \underline{v}\right)=Q(\underline{v}) \quad \forall \underline{v} \in \mathcal{U}_{0, h},
$$

where $\mathcal{U}_{0, h}=\left\{\underline{v} \in \mathcal{U}_{B, h} \mid \mathcal{A}_{m}^{h}(\underline{v}, \underline{v})=0\right\}$.

To analyze the discretization errors $e_{M}=||\left|\underline{u}-\underline{u}_{h}\right|||_{M, h}$ and $e_{B}=\left.||\left|\underline{u}-\underline{u}_{h}\right|\right|_{B, h}$ as originating from (3.2) and (3.3) when $t>0$, we split $e_{M}$ and $e_{B}$ into two orthogonal components in both cases, namely the approximation errors

$$
\begin{aligned}
& e_{a, M}(\underline{u})=\left.\min _{\underline{v} \in \mathcal{U}_{M, h}}\|\underline{u}-\underline{v}\|\right|_{M, h}, \\
& e_{a, B}(\underline{u})=\min _{\underline{v} \in \mathcal{U}_{B, h}}\|\underline{u}-\underline{v}\| \|_{B, h},
\end{aligned}
$$

and the consistency errors

$$
\begin{aligned}
e_{c, M}(\underline{u}) & =\sup _{\underline{v} \in \mathcal{U}_{M, h}} \frac{\left(\mathcal{A}_{M}-\mathcal{A}_{M}^{h}\right)(\underline{u}, \underline{v})}{\|\underline{v}\|_{M, h}}, \\
e_{c, B}(\underline{u}) & =\sup _{\underline{v} \in \mathcal{U}_{B, h}} \frac{\left(\mathcal{A}_{B}-\mathcal{A}_{B}^{h}\right)(\underline{u}, \underline{v})}{\|\underline{v}\|_{B, h}},
\end{aligned}
$$

where $\||\cdot|\|_{M, h}=\sqrt{\mathcal{A}_{M}^{h}(\cdot, \cdot)},\||\cdot|\|_{B, h}=\sqrt{\mathcal{A}_{B}^{h}(\cdot, \cdot)}$. These definitions imply that

$$
\begin{aligned}
e_{M}^{2} & =e_{a, M}^{2}+e_{c, M}^{2}, \\
e_{B}^{2} & =e_{a, B}^{2}+e_{c, B}^{2} .
\end{aligned}
$$


(For detailed reasoning, see [6].) We note that standard finite element theory gives the bound $e_{a, M} \leq C h\|\underline{u}\|_{2}$, and for $e_{a, B}$ we refer to [3]. Hence, the main task of this paper is to bound $e_{c, M}$ and $e_{c, B}$. We aim to analyze these error terms with both proposed strain-reductions (E1) and (E2).

The asymptotic formulations (2.5), (3.4) lead to a similar error decomposition. We have for $\underline{u}_{0} \in \mathcal{U}_{0}$ the asymptotic approximation error

$$
e_{a}^{0}\left(\underline{u}_{0}\right)=\min _{\underline{v} \in \mathcal{U}_{0, h}}\left|\left\|\underline{u}_{0}-\underline{v} \mid\right\|_{B, h},\right.
$$

which was under consideration in [3]. On the other hand, at $t=0$ we have that

$$
\mathcal{A}_{b}\left(\underline{u}_{0}, \underline{v}\right)=Q(\underline{v}) \quad \forall \underline{v} \in \mathcal{U}_{0}
$$

for the inextensional solution $\underline{u}_{0} \in \mathcal{U}_{0}$, and that

$$
\mathcal{A}_{b}\left(\underline{u}_{h}, \underline{v}\right)=Q(\underline{v}) \quad \forall \underline{v} \in \mathcal{U}_{0, h}
$$

for the corresponding finite element solution $\underline{u}_{h}$. Let $\underline{\tilde{u}}_{h}$ be the best finite element approximation to $\underline{u}_{0}$ in $\mathcal{U}_{0, h}$, i.e.

$$
\mathcal{A}_{b}\left(\underline{\tilde{x}}_{h}, \underline{v}\right)=\mathcal{A}_{b}\left(\underline{u}_{0}, \underline{v}\right) \quad \forall \underline{v} \in \mathcal{U}_{0, h} .
$$

By (3.7), (3.8) the asymptotic consistency error $\underline{u}_{h}-\underline{\tilde{u}}_{h} \in \mathcal{U}_{0, h}$ satisfies

$$
\mathcal{A}_{b}\left(\underline{u}_{h}-\underline{\tilde{u}}_{h}, \underline{v}\right)=Q(\underline{v})-\mathcal{A}_{b}\left(\underline{u}_{0}, \underline{v}\right) \quad \forall \underline{v} \in \mathcal{U}_{0, h},
$$

and thus we can define

$$
e_{c}^{0}\left(\underline{u}_{0}\right) \doteqdot\left|\left\|\underline{u}_{h}-\underline{\tilde{u}}_{h} \mid\right\|_{h}=\sup _{\underline{v} \in \mathcal{U}_{0, h}} \frac{Q(\underline{v})-\mathcal{A}_{b}\left(\underline{u}^{0}, \underline{v}\right)}{\|\underline{v} \mid\|_{B, h}} .\right.
$$

As in [3, the main tool of our analysis will be the Fourier transform, where we write

$$
\begin{gathered}
\underline{u}(x, y)=\sum_{\lambda \in \Lambda} \varphi_{\lambda}(y) \underline{\phi}_{\lambda}(x)=\sum_{\lambda \in \Lambda} \underline{\vartheta}_{\lambda}(x, y), \\
\varphi_{\lambda}(y)=e^{i \lambda y}, \quad \Lambda=\left\{\lambda=\frac{2 \pi \nu}{H}, \nu \in \mathbb{Z}\right\},
\end{gathered}
$$

making use of the periodic boundary conditions at $y=0, H$. For functions in the FE space we write analogously

$$
\underline{v}(x, y)=\sum_{\lambda \in \Lambda_{N}} \tilde{\varphi}_{\lambda}(y) \tilde{\Phi}_{\lambda}(x)=\sum_{\lambda \in \Lambda_{N}} \underline{\tilde{\vartheta}}_{\lambda}(x, y),
$$

where

$$
\begin{gathered}
\Lambda_{N}=\left\{\lambda \in \Lambda \mid-\pi \leq \lambda h_{y} \leq \pi \text { when } N_{y}\right. \text { is odd } \\
\text { or } \left.-\pi<\lambda h_{y} \leq \pi \text { when } N_{y} \text { is even }\right\}
\end{gathered}
$$

Here $\tilde{\varphi}_{\lambda}(y)$ is the interpolant of $\varphi_{\lambda}(y)$, so that we are in fact considering a discrete Fourier transform of $\underline{v} \in \mathcal{U}_{h}$.

In our forthcoming analysis the following results are also needed. 
Proposition 3.1 (Korn's inequality). Let $\mathcal{V}=\left\{\underline{v}=\left(v_{1}, v_{2}\right) \in\left[H_{p}^{1}(\Omega)\right]^{2} \mid \underline{v}(0, \cdot)=\right.$ $\underline{v}(L, \cdot)=0\}$ or let $\mathcal{V}=\left\{\underline{v}=\left(v_{1}, v_{2}\right) \in\left[H_{p}^{1}(\Omega)\right]^{2} \mid \int_{\Omega} v_{1} d x d y=\int_{\Omega} v_{2} d x d y=0\right\}$. Then there exists a constant $c>0$ such that

$$
\|\underline{v}\|_{1} \leq c\left(\left\|\frac{\partial v_{1}}{\partial x}\right\|_{L^{2}}^{2}+\left\|\frac{\partial v_{2}}{\partial y}\right\|_{L^{2}}^{2}+2\left\|\frac{1}{2}\left(\frac{\partial v_{1}}{\partial y}+\frac{\partial v_{2}}{\partial x}\right)\right\|_{L^{2}}^{2}\right)^{1 / 2} \quad \forall \underline{v} \in \mathcal{V} .
$$

Proof. See [2].

Proposition 3.2. Assume that $\underline{v}=\left(v_{1}, v_{2}\right) \in\left[\mathcal{V}_{h}\right]^{2}$. Then

$$
\left\|\frac{\partial v_{1}}{\partial y}+\frac{\partial v_{2}}{\partial x}\right\|_{L^{2}} \leq C\left(\left\|\Pi_{h}^{x y}\left(\frac{\partial v_{1}}{\partial y}+\frac{\partial v_{2}}{\partial x}\right)\right\|_{L^{2}}+\left\|\frac{\partial v_{1}}{\partial x}\right\|_{L^{2}}+\left\|\frac{\partial v_{2}}{\partial y}\right\|_{L^{2}}\right) .
$$

Proof. See Theorem 6.1 in 6 .

\section{The COnSistency ERRor at $t>0$}

We start by giving a stability result for $\mathcal{U}_{M, h}$.

Lemma 4.1. Let $\underline{v} \in \mathcal{U}_{M, h}$. Then

$$
\|\underline{v}\|_{1} \leq C t^{-1}\left|\|\underline{v} \mid\|_{M, h} .\right.
$$

Proof. Assume (E1) first. By (3.2) we have that for $\underline{v}=(u, v, w, \theta, \psi) \in \mathcal{U}_{M, h}$

$$
\left\|\frac{\partial \theta}{\partial x}\right\|_{L^{2}}+\left\|\frac{\partial \psi}{\partial y}\right\|_{L^{2}}+\left\|\frac{\partial \theta}{\partial y}+\frac{\partial \psi}{\partial x}\right\|_{L^{2}} \leq C t^{-1} \mid\|\underline{v}\|_{M, h}
$$

and thus, by the Korn inequality (3.11),

$$
\|\theta\|_{1}+\|\psi\|_{1} \leq C t^{-1} \mid\|\underline{v}\| \|_{M, h}
$$

Also the definitions (2.1) of the membrane strains $\beta_{i j}$ imply

$$
\left\|\frac{\partial u}{\partial x}\right\|_{L^{2}}+\left\|\frac{\partial v}{\partial y}\right\|_{L^{2}}+\left\|\Pi_{h}^{x y}\left(\frac{\partial u}{\partial y}+\frac{\partial v}{\partial x}\right)\right\|_{L^{2}} \leq C\left(\|\underline{\|}\|\left\|_{M, h}+\right\| w \|_{L^{2}}\right),
$$

and by (3.12) we have

$$
\left\|\frac{\partial u}{\partial y}+\frac{\partial v}{\partial x}\right\|_{L^{2}} \leq C\left(\left\|\Pi_{h}^{x y}\left(\frac{\partial u}{\partial y}+\frac{\partial v}{\partial x}\right)\right\|_{L^{2}}+\left\|\frac{\partial u}{\partial x}\right\|_{L^{2}}+\left\|\frac{\partial v}{\partial y}\right\|_{L^{2}}\right),
$$

resulting in

$$
\left\|\frac{\partial u}{\partial x}\right\|_{L^{2}}+\left\|\frac{\partial v}{\partial y}\right\|_{L^{2}}+\left\|\frac{\partial u}{\partial y}+\frac{\partial v}{\partial x}\right\|_{L^{2}} \leq C\left(\|\underline{v}\|\left\|_{M, h}+\right\| w \|_{L^{2}}\right),
$$

where, again from by the Korn inequality (3.11),

$$
\|u\|_{1}+\|v\|_{1} \leq C\left(\|\underline{v} \mid\|_{M, h}+\|w\|_{L^{2}}\right) .
$$


By (2.2), (3.1) we have that $\frac{\partial w}{\partial x}=\tilde{\rho}_{1}-\Pi_{h}^{x} \theta$ and $\frac{\partial w}{\partial y}=\tilde{\rho}_{2}-\Pi_{h}^{y} \psi$, so that

$$
\left\|\frac{\partial w}{\partial x}\right\|_{L^{2}}+\left\|\frac{\partial w}{\partial y}\right\|_{L^{2}} \leq C\left(\|\underline{v}\|\left\|_{M, h}+\right\| \theta\left\|_{L^{2}}+\right\| \psi \|_{L^{2}}\right) \leq C t^{-1}|\|\underline{v}\||_{M, h}
$$

by (4.1). The claim follows from (4.1) - (4.3) together with Poincaré's inequality. Similar calculations imply the result also for the modification (E2).

Lemma 4.2. Let $\underline{v} \in \mathcal{U}_{B, h}$. Then

$$
\|\underline{v}\|_{1} \leq C\left|\|\underline{v} \mid\|_{B, h}\right.
$$

Proof. By the definition of $\mathcal{U}_{B, h}$ the Korn inequality (3.11) holds for the pairs $(\theta, \psi)$ and $(u, v)$, as well as Poincaré's inequality for $w$. The result follows as in Lemma 4.1.

Next we derive more specific stability results for the low-order discrete Fourier modes in the FE space.

Lemma 4.3. Let $\underline{\tilde{\vartheta}}_{\lambda}=\tilde{\varphi}_{\lambda} \tilde{\phi}_{\lambda}=\tilde{\varphi}_{\lambda}\left(\tilde{u}_{\lambda}, \tilde{v}_{\lambda}, \tilde{w}_{\lambda}, \tilde{\theta}_{\lambda}, \tilde{\psi}_{\lambda}\right) \in \mathcal{U}_{M, h}$. Then, if $b \neq 0$, we have, for $\lambda \neq 0$ such that $\mid \overline{\lambda \mid} h \leq c<\pi$,

$$
\left\|\tilde{\varphi}_{\lambda} \tilde{u}_{\lambda}\right\|_{1}+\left\|\tilde{\varphi}_{\lambda} \tilde{v}_{\lambda}\right\|_{1}+\left\|\tilde{\varphi}_{\lambda} \tilde{w}_{\lambda}\right\|_{L^{2}} \leq\left. C|\lambda|^{1-m}||\left|\tilde{\tilde{q}}_{\lambda}\right|\right|_{M, h},
$$

where $m=1$ in the elliptic case and $m=0$ in the parabolic and hyperbolic cases, and for $\lambda=0$

$$
\left\|\tilde{\varphi}_{0} \tilde{u}_{0}\right\|_{1}+\left\|\tilde{\varphi}_{0} \tilde{v}_{0}\right\|_{1}+\left\|\tilde{\varphi}_{0} \tilde{w}_{0}\right\|_{L^{2}} \leq C\left|\left\|\underline{\hat{\vartheta}}_{0}\right\|\right|_{M, h}
$$

in any geometry.

Proof. Consider first the case (E1). The translation components $\tilde{u}_{\lambda}$ and $\tilde{v}_{\lambda}$ of $\underline{\tilde{\vartheta}}_{\lambda}$ satisfy the difference equation (cf. [3]

$$
\left(\begin{array}{c}
\tilde{v}_{\lambda} \\
\tilde{u}_{\lambda}
\end{array}\right)\left(x^{k+1}\right)-\left(\begin{array}{c}
\tilde{v}_{\lambda} \\
\tilde{u}_{\lambda}
\end{array}\right)\left(x^{k}\right)=\frac{1}{2} \tau_{k} M\left[\left(\begin{array}{c}
\tilde{v}_{\lambda} \\
\tilde{u}_{\lambda}
\end{array}\right)\left(x^{k+1}\right)+\left(\begin{array}{c}
\tilde{v}_{\lambda} \\
\tilde{u}_{\lambda}
\end{array}\right)\left(x^{k}\right)\right]+h_{x}^{k} \tilde{F}_{\lambda}^{k},
$$

where $\tau_{k}=2 \frac{h_{x}^{k}}{h_{y}} \tan \left(\frac{1}{2} \lambda h_{y}\right)$,

$$
M=i\left(\begin{array}{cc}
\frac{2 c}{b} & -1 \\
\frac{a}{b} & 0
\end{array}\right)
$$

and

$$
\tilde{F}_{\lambda}^{k}=\frac{1}{\cos \left(\frac{1}{2} \lambda h_{y}\right)}\left(\begin{array}{c}
2 \tilde{f}_{12}^{\lambda}\left(x^{k+1 / 2}\right)-\frac{c}{b}\left(\tilde{f}_{22}^{\lambda}\left(x^{k+1}\right)+\tilde{f}_{22}^{\lambda}\left(x^{k}\right)\right) \\
\cos \left(\frac{1}{2} \lambda h_{y}\right) \tilde{f}_{11}^{\lambda}\left(x^{k+1 / 2}\right)-\frac{a}{2 b}\left(\tilde{f}_{22}^{\lambda}\left(x^{k+1}\right)+\tilde{f}_{22}^{\lambda}\left(x^{k}\right)\right)
\end{array}\right) .
$$

Here

$$
\begin{aligned}
\tilde{f}_{11}^{\lambda}\left(x^{k+1 / 2}\right) & =e^{-\left.i n \lambda h_{y}\left(\tilde{\beta}_{11}\left(\underline{\tilde{\vartheta}}_{\lambda}\right)\right)\right|_{\left(x^{k+1 / 2}, y^{n}\right)},}, \\
\tilde{f}_{22}^{\lambda}\left(x^{k}\right) & =e^{-\left.i(n+1 / 2) \lambda h_{y}\left(\tilde{\beta}_{22}\left(\underline{\tilde{\vartheta}}_{\lambda}\right)\right)\right|_{\left(x^{k}, y^{n+1 / 2}\right)},} \\
\tilde{f}_{12}^{\lambda}\left(x^{k+1 / 2}\right) & =\left.e^{-i(n+1 / 2) \lambda h_{y}}\left(\tilde{\beta}_{12}\left(\underline{\tilde{\vartheta}}_{\lambda}\right)\right)\right|_{\left(x^{k+1 / 2}, y^{n+1 / 2}\right)}
\end{aligned}
$$


Due to the constraints at $x=0, L$, we may without loss of generality consider only the exponentially decreasing solution of (4.6) starting from $x=0$. Then if $|\lambda| h_{y} \leq c<\pi$, the standard theory for A-stable difference schemes (see also [3]) gives us the bound

$$
\begin{aligned}
\left\|\left(\begin{array}{c}
\tilde{v}_{\lambda} \\
\tilde{u}_{\lambda}
\end{array}\right)\left(x^{k+1}\right)\right\| \leq & e^{-\alpha|\lambda| x^{k+1}}\left\|\left(\begin{array}{c}
\tilde{v}_{\lambda} \\
\tilde{u}_{\lambda}
\end{array}\right)(0)\right\| \\
& +\int_{0}^{x^{k+1}} e^{-\beta|\lambda|\left(x^{k+1}-t\right)}\left\|\tilde{F}_{\lambda}(t)\right\| e^{-\alpha|\lambda| t} d t
\end{aligned}
$$

where $\|\cdot\|$ is the Euclidean norm of vectors in $\mathbb{R}^{2}$ and

$$
\tilde{F}_{\lambda}=\left(\begin{array}{c}
2 \tilde{f}_{12}^{\lambda}-\frac{2 c}{b} \tilde{f}_{22}^{\lambda} \\
\tilde{f}_{11}^{\lambda}-\frac{a}{b} \tilde{f}_{22}^{\lambda}
\end{array}\right)
$$

Here $\alpha>\beta>0$ in the elliptic case and $\alpha=\beta=0$ in the parabolic and hyperbolic cases. Since $\tilde{u}_{\lambda}(0)=\tilde{v}_{\lambda}(0)=0$, we obtain, when $\lambda \neq 0$,

$\left\|\left(\begin{array}{c}\tilde{v}_{\lambda} \\ \tilde{u}_{\lambda}\end{array}\right)\left(x^{k+1}\right)\right\|^{2} \leq\left\{\begin{array}{l}C|\lambda|^{-1} e^{-2 \beta|\lambda| x^{k+1}} \int_{0}^{x^{k+1}}\left\|\tilde{F}_{\lambda}(t)\right\|^{2} d t \text { in the elliptic case, } \\ C \int_{0}^{x^{k+1}}\left\|\tilde{F}_{\lambda}(t)\right\|^{2} d t \text { in the parabolic and hyperbolic cases, }\end{array}\right.$

and consequently

$$
\left\|\tilde{v}_{\lambda}\right\|_{L^{2}(0, L)}^{2}+\left\|\tilde{u}_{\lambda}\right\|_{L^{2}(0, L)}^{2} \leq C|\lambda|^{-2 m}\left\|\tilde{F}_{\lambda}\right\|_{L^{2}(0, L)}^{2} .
$$

Also, (4.6) gives the relation

$$
\left(\begin{array}{c}
\tilde{v}_{\lambda} \\
\tilde{u}_{\lambda}
\end{array}\right)^{\prime}\left(x^{k+1 / 2}\right)=\frac{1}{h_{y}} \tan \left(\frac{1}{2} \lambda h_{y}\right) M\left[\left(\begin{array}{c}
\tilde{v}_{\lambda} \\
\tilde{u}_{\lambda}
\end{array}\right)\left(x^{k+1}\right)+\left(\begin{array}{c}
\tilde{v}_{\lambda} \\
\tilde{u}_{\lambda}
\end{array}\right)\left(x^{k}\right)\right]+\tilde{F}_{\lambda}^{k},
$$

from which it follows that

$$
\begin{aligned}
& \left\|\tilde{v}_{\lambda}^{\prime}\right\|_{L^{2}(0, L)}^{2}+\left\|\tilde{u}_{\lambda}^{\prime}\right\|_{L^{2}(0, L)}^{2} \\
& \quad \leq C|\lambda|^{2}\left(\left\|\tilde{v}_{\lambda}\right\|_{L^{2}(0, L)}^{2}+\left\|\tilde{u}_{\lambda}\right\|_{L^{2}(0, L)}^{2}\right)+\left\|\tilde{F}_{\lambda}\right\|_{L^{2}(0, L)}^{2} \\
& \quad \leq C|\lambda|^{2(1-m)}\left\|\tilde{F}_{\lambda}\right\|_{L^{2}(0, L)}^{2} .
\end{aligned}
$$

Combining 4.10 and 4.11) gives

$$
\left\|\tilde{\varphi}_{\lambda} \tilde{u}_{\lambda}\right\|_{1}+\left\|\tilde{\varphi}_{\lambda} \tilde{v}_{\lambda}\right\|_{1} \leq C|\lambda|^{1-m}||\left|\underline{\tilde{\vartheta}}_{\lambda}\right| \|_{M, h}
$$

since $\left\|\tilde{F}_{\lambda}\right\|_{L^{2}} \leq C\|\| \underline{\tilde{\vartheta}}_{\lambda}\|\|_{M, h}$.

To consider $\tilde{w}_{\lambda}$, we note that (cf. [3])

and thus

$$
\tilde{w}_{\lambda}\left(x^{k}\right)=\frac{-2 i}{b h_{y}} \tan \left(\frac{1}{2} \lambda h_{y}\right) \tilde{v}_{\lambda}\left(x^{k}\right)+\frac{1}{b \cos \left(\frac{1}{2} \lambda h_{y}\right)} \tilde{f}_{22}^{\lambda}\left(x^{k}\right),
$$

leading to

$$
\left\|\tilde{w}_{\lambda}\right\|_{L(0, L)}^{2} \leq C\left(|\lambda|^{2}\left\|\tilde{v}_{\lambda}\right\|_{L^{2}(0, L)}^{2}+\left\|\tilde{f}_{22}^{\lambda}\right\|_{L^{2}(0, L)}^{2}\right)
$$

The claim for $\lambda \neq 0$ follows from (4.12) together with (4.13).

When $\lambda=0$ we have from (4.9) and from $\tilde{w}_{0}\left(x^{k}\right)=\frac{1}{b} \tilde{f}_{22}\left(x^{k}\right)$ that

$$
\left\|\tilde{\varphi}_{0} \tilde{u}_{0}\right\|_{1}+\left\|\tilde{\varphi}_{0} \tilde{v}_{0}\right\|_{1}+\left\|\tilde{\varphi}_{0} \tilde{w}_{0}\right\|_{L^{2}} \leq C\left\|\mid \underline{\tilde{\vartheta}}_{0}\right\| \|_{M, h}
$$


regardless of geometry. Similar calculations show that the claim holds also for the case (E2).

Remark 4.1. The assumption $b \neq 0$ is not superfluous. This can be seen by taking $b=0, \lambda=0, a=-1, c=1 / 2$ and choosing $\tilde{\phi}_{0}\left(x^{1}\right)=(0,0,2,4 / h, 0)$, then repeating the sequence $\tilde{\phi}_{0}\left(x^{j}\right)=(h,-h, 0,-8 / h, 0), \underline{\tilde{\phi}}_{0}\left(x^{j+1}\right)=(0,0,-2,4 / h, 0), \underline{\phi}_{0}\left(x^{j+2}\right)=$ $(-h, h, 0,0,0), \underline{\phi}_{0}\left(x^{j+3}\right)=(0,0,2,4 / h, 0)$ for $j=2,6,10, \ldots$, and finally letting $\underline{\tilde{\phi}}_{0}\left(x^{N_{x}-2}\right)=(h,-h, 0,-8 / h, 0), \underline{\phi}_{0}\left(x^{N_{x}-1}\right)=(0,0,-2,4 / h, 0)$. For this particular choice we have that $\left\|\frac{\partial \tilde{u}_{0}}{\partial x}\right\|_{L^{2}} \sim \min \left\{\frac{1}{h}, \frac{h^{2}}{t}\right\} \mid\left\|\underline{\tilde{\vartheta}}_{0}\right\| \|_{M, h}$, so the stability is weaker when $b=0$.

With the help of the stability estimates given in Lemmas $4.1-4.3$ we can now bound the consistency error.

Theorem 4.4. Assume that $b \neq 0$, and let $m=1$ in the elliptic case and $m=0$ in the parabolic and hyperbolic cases. The consistency error $e_{c, M}$ defined in (3.5) satisfies

$$
e_{c, M} \leq C_{1}(\underline{u}) h+C_{2}(t, \underline{u}) h^{2}+C_{3}(t, s, \underline{u}) h^{1+s}+C_{4}(t, \underline{u}) h^{2}, \quad s \geq 0,
$$

provided that

$$
\begin{aligned}
C_{1}(\underline{u}) & =C \sum_{i j}\left|\beta_{i j}(\underline{u})\right|_{2-m}, \\
C_{2}(\underline{u}, t) & =\left\{\begin{array}{l}
0 \text { for the case }(E 1), \\
C t^{-1}|w|_{1} \text { for the case (E2), }
\end{array}\right. \\
C_{3}(t, s, \underline{u}) & =C t^{-1} \sum_{i}\left|\beta_{i i}(\underline{u})\right|_{1+s}+\left\{\begin{array}{l}
C t^{-1}\left|\beta_{12}(\underline{u})\right|_{1+s} \text { for the case (E1), } \\
C t^{-1}\left(\left|\beta_{12}(\underline{u})\right|_{s}+|w|_{1+s}\right) \text { for the case (EQ), }
\end{array}\right. \\
C_{4}(t, \underline{u}) & =C t^{-1}\left(\sum_{i}\left|\rho_{i}(\underline{u})\right|_{1}\right)
\end{aligned}
$$

are all finite. The consistency error $e_{c, B}$ defined in (3.6) satisfies

$$
e_{c, B} \leq C_{1}(t, \underline{u}) h+C_{2}(t, \underline{u}) h^{2},
$$

provided that

$$
C_{1}(t, \underline{u})=C t^{-2} \sum_{i j}\left|\beta_{i j}(\underline{u})\right|_{1}
$$

and

$$
C_{2}(t, \underline{u})=C t^{-2} \sum_{i}\left|\rho_{i}(\underline{u})\right|_{1}
$$

are both finite.

Remark 4.2. The transverse shear strains $\rho_{i}$ are typically very small at small $t$ in smooth deformation states (see, e.g., [5]), so the error term of $e_{c, M}$ is very likely negligible in practice. In the bending-dominated case, $e_{c, B}$ depends strongly on $\beta_{i j}$ and $\rho_{i}$. For smooth deformations one could assume realistically that $\left|\beta_{i j}(\underline{u})\right|_{1} \sim$ $\left|\rho_{i}(\underline{u})\right|_{1} \sim t^{2}$ as $t \rightarrow 0$, in which case $e_{c, B}=O(h)$ uniformly in $t$. In practice, however, boundary layer effects probably cause the growth of $e_{c, B}$, via constant $C_{1}(\underline{u})$ in particular. 
Proof. We consider first the membrane case, and write $\underline{u}=\sum_{\lambda \in \Lambda} \underline{\vartheta}_{\lambda} \in \mathcal{U}_{M}$ and $\underline{v}=\sum_{\lambda \in \Lambda_{N}} \underline{\tilde{\vartheta}}_{\lambda} \in \mathcal{U}_{M, h}$. Then by the orthogonality of the discrete and continuous modes (cf. [3])

$$
\begin{aligned}
\left(\mathcal{A}_{M}-\right. & \left.\mathcal{A}_{M}^{h}\right)(\underline{u}, \underline{v})=\left(\mathcal{A}_{m}-\mathcal{A}_{m}^{h}\right)(\underline{u}, \underline{v})=\left(\mathcal{A}_{m}-\mathcal{A}_{m}^{h}\right)\left(\sum_{\lambda \in \Lambda} \underline{\vartheta}_{\lambda} \sum_{\lambda \in \Lambda_{N}} \underline{\tilde{\vartheta}}_{\lambda}\right) \\
= & \left(\mathcal{A}_{m}-\mathcal{A}_{m}^{h}\right)\left(\sum_{|\lambda| \leq \lambda_{0}} \underline{\vartheta}_{\lambda}, \sum_{\lambda \in \Lambda_{N}} \underline{\tilde{\vartheta}}_{\lambda}\right)+\left(\mathcal{A}_{m}-\mathcal{A}_{m}^{h}\right)\left(\sum_{|\lambda|>\lambda_{0}} \underline{\vartheta}_{\lambda}, \sum_{\lambda \in \Lambda_{N}} \underline{\tilde{\vartheta}}_{\lambda}\right) \\
= & \sum_{|\lambda| \leq \lambda_{0}}\left(\mathcal{A}_{m}-\mathcal{A}_{m}^{h}\right)\left(\underline{\vartheta}_{\lambda}, \underline{\tilde{\vartheta}}_{\lambda}\right)+\sum_{|\lambda|>\lambda_{0}}\left(\mathcal{A}_{m}-\mathcal{A}_{m}^{h}\right)\left(\underline{\vartheta}_{\lambda}, \underline{v}\right) \\
\leq & C \sum_{i j} \sum_{|\lambda| \leq \lambda_{0}}\left[\left(\bar{\beta}_{i j}\left(\underline{\vartheta}_{\lambda}\right), \beta_{i j}\left(\underline{\tilde{\vartheta}}_{\lambda}\right)\right)-\left(\tilde{\tilde{\beta}}_{i j}\left(\underline{\vartheta}_{\lambda}\right), \tilde{\beta}_{i j}\left(\underline{\tilde{\vartheta}}_{\lambda}\right)\right)\right] \\
& \left.+\sum_{i j, i \neq j|\lambda| \leq \lambda_{0}}\left[\left(\bar{\beta}_{i i} \underline{\vartheta}_{\lambda}\right), \beta_{j j}\left(\underline{\tilde{\vartheta}}_{\lambda}\right)\right)-\left(\overline{\tilde{\beta}}_{i i}\left(\underline{\vartheta}_{\lambda}\right), \tilde{\beta}_{j j}\left(\underline{\tilde{\vartheta}}_{\lambda}\right)\right)\right] \\
& +C\left|\lambda_{0}\right|^{-s_{1}} \sum_{i} \sum_{|\lambda|>\lambda_{0}}|\lambda|^{s_{1}} \mid\left(\bar{\beta}_{i i}\left(\underline{\vartheta}_{\lambda}\right)-\overline{\tilde{\beta}}_{i i}\left(\underline{\vartheta}_{\lambda}\right), \beta_{i i}(\underline{v})-\tilde{\beta}_{i i}\left(\underline{v}_{)}\right) \mid\right. \\
& +C\left|\lambda_{0}\right|^{-s_{2}} \sum_{i \neq j} \sum_{|\lambda|>\lambda_{0}}|\lambda|^{s_{2}} \mid\left(\bar{\beta}_{i i}\left(\underline{\vartheta}_{\lambda}\right)-\Pi_{h}^{x y} \bar{\beta}_{i i}\left(\underline{\vartheta}_{\lambda}\right), \beta_{j j}\left(\underline{v}_{)}\right) \mid\right. \\
& +C\left|\lambda_{0}\right|^{-s_{3}} \sum_{|\lambda|>\lambda_{0}}|\lambda|^{s_{3}} \mid \int_{\Omega}\left(\overline { \beta } _ { 1 2 } ( \underline { \vartheta } _ { \lambda } ) \beta _ { 1 2 } \left(\underline{v}_{)}-\overline{\tilde{\beta}}_{12}\left(\underline{\vartheta}_{\lambda}\right) \tilde{\beta}_{12}\left(\underline{v}_{)}\right) d x d y \mid\right.\right. \\
& +C \sum_{i} \sum_{\lambda \in \Lambda}\left(\bar{\rho}_{i}\left(\underline{\vartheta}_{\lambda}\right)-\overline{\tilde{\rho}}_{i}\left(\underline{\vartheta}_{\lambda}\right), \rho_{i}(\underline{v})-\tilde{\rho}_{i}\left(\underline{v}_{)}\right)\right. \\
= & I+I I+I I I+I V+V+V I,
\end{aligned}
$$

where we have chosen $\lambda_{0}$ such that $\lambda_{0} h \leq c<\pi$. We note first that in $I$

$$
\begin{aligned}
& \left(\bar{\beta}_{i j}\left(\underline{\vartheta}_{\lambda}\right), \beta_{i j}\left(\underline{\tilde{\vartheta}}_{\lambda}\right)\right)-\left(\overline{\tilde{\beta}}_{i j}\left(\underline{\vartheta}_{\lambda}\right), \tilde{\beta}_{i j}\left(\underline{\tilde{\vartheta}}_{\lambda}\right)\right) \\
& \quad=\left(\bar{\beta}_{i j}\left(\underline{\vartheta}_{\lambda}\right)-\overline{\tilde{\beta}}_{i j}\left(\underline{\vartheta}_{\lambda}\right), \beta_{i j}\left(\tilde{\tilde{\vartheta}}_{\lambda}\right)\right)+\left(\overline{\tilde{\beta}}_{i j}\left(\underline{\vartheta}_{\lambda}\right), \beta_{i j}\left(\underline{\tilde{\vartheta}}_{\lambda}\right)-\tilde{\beta}_{i j}\left(\underline{\tilde{\vartheta}}_{\lambda}\right)\right) .
\end{aligned}
$$

Here the first term can be bounded as

$$
\begin{aligned}
\left(\bar{\beta}_{i j}\left(\underline{\vartheta}_{\lambda}\right)-\overline{\tilde{\beta}}_{i j}\left(\underline{\vartheta}_{\lambda}\right), \beta_{i j}\left(\tilde{\vartheta}_{\lambda}\right)\right) & \leq\left.\left\|\beta_{i j}\left(\underline{\vartheta}_{\lambda}\right)-\tilde{\beta}_{i j}\left(\underline{\vartheta}_{\lambda}\right)\right\|\right|_{L^{2}}\left\|\beta_{i j}\left(\tilde{\vartheta}_{\lambda}\right)\right\|_{L^{2}} \\
& \leq C h\left|\beta_{i j}\left(\underline{\vartheta}_{\lambda}\right)\right|_{1}\left(\left\|\tilde{\varphi}_{\lambda} \tilde{u}_{\lambda}\right\|_{1}+\left\|\tilde{\varphi}_{\lambda} \tilde{v}_{\lambda}\right\|_{1}+\left\|\tilde{\varphi}_{\lambda} \tilde{w}_{\lambda}\right\|_{L^{2}}\right) \\
& \leq\left. C h\left|\beta_{i j}\left(\underline{\vartheta}_{\lambda}\right)\right|_{1}|\lambda|^{1-m}|| \underline{\tilde{\vartheta}}_{\lambda}||\right|_{M, h} \\
& \leq\left. C h\left|\beta_{i j}\left(\underline{\vartheta}_{\lambda}\right)\right|_{2-m}\left|\| \underline{\tilde{\vartheta}}_{\lambda}\right|\right|_{M, h},
\end{aligned}
$$

where the third inequality follows from Lemma 4.3 and the last inequality from the fact that

$$
\frac{\partial}{\partial y} \beta_{i j}\left(\vartheta_{\lambda}\right)=i \lambda \beta_{i j}\left(\vartheta_{\lambda}\right)
$$


The second term in (4.15) is zero in the case of the modification (E1), since $R^{i j}$ is an orthogonal $L^{2}$-projection. For the modification (E2) the second term gives

$$
\begin{aligned}
& \left(\overline{\tilde{\beta}}_{i j}\left(\underline{\vartheta}_{\lambda}\right), \beta_{i j}\left(\underline{\tilde{\vartheta}}_{\lambda}\right)-\tilde{\beta}_{i j}\left(\underline{\tilde{\vartheta}}_{\lambda}\right)\right) \\
& =\left(\overline{\tilde{\beta}}_{12}\left(\underline{\vartheta}_{\lambda}\right),-S_{12}\left(\underline{\tilde{\vartheta}}_{\lambda}\right)\right) \\
& =\left(\bar{\beta}_{12}\left(\underline{\vartheta}_{\lambda}\right),-S_{12}\left(\underline{\tilde{\vartheta}}_{\lambda}\right)\right)-\left(\bar{S}_{12}\left(\underline{\vartheta}_{\lambda}\right), S_{12}\left(\underline{\tilde{\vartheta}}_{\lambda}\right)\right) \\
& \leq\left\|a \frac{\partial}{\partial y} \bar{\beta}_{12}\left(\underline{\vartheta}_{\lambda}\right)+b \frac{\partial}{\partial x} \bar{\beta}_{12}\left(\underline{\vartheta}_{\lambda}\right)\right\|_{L^{2}} h\left\|\tilde{\varphi}_{\lambda} \tilde{w}_{\lambda}\right\|_{L^{2}} \\
& +\left\|c\left(I-\Pi_{h}^{x y}\right) \bar{\beta}_{12}\left(\underline{\vartheta}_{\lambda}\right)\right\|_{L^{2}}\left\|\tilde{\varphi}_{\lambda} \tilde{w}_{\lambda}\right\|_{L^{2}} \\
& +C h\left|\varphi_{\lambda} w_{\lambda}\right|{ }_{1} h\left|\tilde{\varphi}_{\lambda} \tilde{w}_{\lambda}\right|_{1} \\
& \leq C h\left|\beta_{12}\left(\underline{\vartheta}_{\lambda}\right)\right|_{1}\left\|\tilde{\varphi}_{\lambda} \tilde{w}_{\lambda}\right\|_{L^{2}} \\
& +C h^{2}\left|\varphi_{\lambda} w_{\lambda}\right|_{1}\left|\tilde{\varphi}_{\lambda} \tilde{w}_{\lambda}\right|_{1} \\
& \leq C h\left|\beta_{12}\left(\underline{\vartheta}_{\lambda}\right)\right|_{1}|\lambda|^{m-1}||\left|\underline{\tilde{\vartheta}}_{\lambda}\right|||_{M, h} \\
& +C h^{2} t^{-1}\left|\varphi_{\lambda} w_{\lambda}\right|_{1}||\left|\underline{\tilde{g}}_{\lambda}\right|||_{M, h} \\
& \leq C h\left|\beta_{12}\left(\underline{\vartheta}_{\lambda}\right)\right|_{2-m}||\left|\underline{\tilde{\vartheta}}_{\lambda}\right|||_{M, h}+C h^{2} t^{-1}\left|\varphi_{\lambda} w_{\lambda}\right|_{1}||\left|\underline{\tilde{\vartheta}}_{\lambda}\right|||_{M, h},
\end{aligned}
$$

where the next to last inequality follows from Lemmas 4.1 and 4.3 For the term II we can write

$$
\begin{aligned}
& \left(\bar{\beta}_{i i}\left(\underline{\vartheta}_{\lambda}\right), \beta_{j j}\left(\underline{\tilde{\vartheta}}_{\lambda}\right)\right)-\left(\overline{\tilde{\beta}}_{i i}\left(\underline{\vartheta}_{\lambda}\right), \tilde{\beta}_{j j}\left(\underline{\tilde{\vartheta}}_{\lambda}\right)\right) \\
& \quad=\left(\bar{\beta}_{i i}\left(\underline{\vartheta}_{\lambda}\right)-\overline{\tilde{\beta}}_{i i}\left(\underline{\vartheta}_{\lambda}\right), \beta_{j j}\left(\tilde{\vartheta}_{\lambda}\right)\right)+\left(\overline{\tilde{\beta}}_{i i}\left(\underline{\vartheta}_{\lambda}\right), \beta_{j j}\left(\underline{\tilde{\vartheta}}_{\lambda}\right)-\tilde{\beta}_{j j}\left(\underline{\tilde{\vartheta}}_{\lambda}\right)\right),
\end{aligned}
$$

where the first term can be treated as in case of the term $I$. The second term in (4.18) can be written as

$$
\begin{aligned}
\left(\overline{\tilde{\beta}}_{i i}\left(\underline{\vartheta}_{\lambda}\right), \beta_{j j}\left(\underline{\tilde{\vartheta}}_{\lambda}\right)-\tilde{\beta}_{j j}\left(\underline{\tilde{\vartheta}}_{\lambda}\right)\right) & =\left(R^{i i} \bar{\beta}_{i i}\left(\underline{\vartheta}_{\lambda}\right),\left(I-R^{j j}\right) \beta_{j j}\left(\underline{\tilde{\vartheta}}_{\lambda}\right)\right) \\
& =\left(\left(I-R^{j j}\right) R^{i i} \bar{\beta}_{i i}\left(\underline{\vartheta}_{\lambda}\right), \beta_{j j}\left(\underline{\tilde{\vartheta}}_{\lambda}\right)\right) \\
& \leq C h\left|\beta_{i i}\left(\underline{\vartheta}_{\lambda}\right)\right|_{1}\left(\left\|\tilde{\varphi}_{\lambda} \tilde{u}_{\lambda}\right\|_{1}+\left\|\tilde{\varphi}_{\lambda} \tilde{v}_{\lambda}\right\|_{1}+\left\|\tilde{\varphi}_{\lambda} \tilde{w}_{\lambda}\right\|_{L^{2}}\right) \\
& \leq C h\left|\beta_{i i}\left(\underline{\vartheta}_{\lambda}\right)\right|_{1}|\lambda|^{1-m}\left|\| \underline{\tilde{\vartheta}}_{\lambda}\right|||_{M, h} \\
& \leq C h\left|\beta_{i i}\left(\underline{\vartheta}_{\lambda}\right)\right|_{2-m}\left|\left\|\underline{\tilde{\vartheta}}_{\lambda}\right\|\right|_{M, h},
\end{aligned}
$$

where the next to last inequality is again a direct application of Lemma 4.3. By (4.15) - (4.19) we have the bounds

$$
\begin{aligned}
I+I I \leq & \left.C_{1} h \sum_{i j} \sum_{|\lambda| \leq \lambda_{0}}\left|\beta_{i j}\left(\underline{\vartheta}_{\lambda}\right)\right|_{2-m}||\left|\underline{\tilde{\vartheta}}_{\lambda}\right|\right|_{M, h} \\
& +C_{2} h^{2} t^{-1} \sum_{|\lambda| \leq \lambda_{0}}\left|\varphi_{\lambda} w_{\lambda}\right|_{1}||\left|\underline{\tilde{\vartheta}}_{\lambda}\right|||_{M, h} \\
\leq & C_{1} h \sum_{i j}\left|\beta_{i j}(\underline{u})\right|_{2-m}|||\underline{v}|||_{M, h}+C_{2} h^{2} t^{-1}|w|_{1}|| \underline{\mid} \mid \|_{M, h},
\end{aligned}
$$


where $C_{2}=0$ for the modification (E1). For the rest of the terms in (4.14), standard approximation theory gives

$$
\begin{aligned}
& I I I \leq C h^{2+s_{1}} t^{-1} \sum_{i}\left|\beta_{i i}(\underline{u})\right|_{1+s_{1}}|| \underline{v}||_{M, h}, \\
& I V \leq\left. C h^{1+s_{2}} t^{-1} \sum_{i}\left|\beta_{i i}(\underline{u})\right|_{1+s_{2}}|||\underline{\mid}|\right|_{M, h}, \\
& V \leq\left\{\begin{array}{l}
\left.C h^{1+s_{3}} t^{-1}\left|\beta_{12}(\underline{u})\right|_{1+s_{3}}|||\underline{v}|\right|_{M, h} \text { for the case (E1), } \\
\left.C h^{1+s_{3}} t^{-1}\left(\left|\beta_{12}(\underline{u})\right|_{s_{3}}+|w|_{1+s_{3}}\right)|||\underline{v}|\right|_{M, h} \text { for the case (E2), }
\end{array}\right. \\
& V I \leq C h^{2} t^{-1} \sum_{i}\left|\rho_{i}(\underline{u})\right|_{1}|||\underline{v}| \|_{M, h},
\end{aligned}
$$

by Lemmas 4.1 and 4.3. The claim for $e_{c, M}$ follows form (4.14), (4.20) and (4.21) when we take $s_{1}=s_{2}=s_{3}=s$.

For the case $e_{c, B}$ the claim follows by the same arguments when we note that

$$
\left(\mathcal{A}_{B}-\mathcal{A}_{B}^{h}\right)(\underline{u}, \underline{v})=t^{-2}\left(\mathcal{A}_{m}-\mathcal{A}_{m}^{h}\right)(\underline{u}, \underline{v})
$$

and use the stability result given in Lemma 4.2.

\section{The ASYMPtotic CONSISTENCY ERROR}

In this section we bound the asymptotic consistency error in an inextensional deformation state, as defined by (3.10). In [3] we showed that the approximation error in the inextensional state is of order $O(h)$ under nearly optimal regularity assumptions on $\underline{u}_{0}$. Here we find that the consistency error is likewise of order $O(h)$ at $t=0$, but we need a very strong regularity assumption on $\underline{u}_{0}$.

We also make the additional assumption that the load is given by

$$
Q(\underline{v})=\int_{\Omega}\left(q_{1} u+q_{2} v+q_{3} w\right) d x d y
$$

for some suitable $q_{i} \in L_{p}^{2}(\Omega), i=1,2,3$, where $L_{p}^{2}(\Omega)$ denotes the usual $L^{2}$-space with periodic boundary conditions imposed at $y=0, H$, and we define the Fourier components of the load by

$$
Q^{\lambda}(\underline{v})=\int_{\Omega}\left(q_{1}^{\lambda} u+q_{2}^{\lambda} v+q_{3}^{\lambda} w\right) d x d y
$$

where for each $q_{i}$ we write $q_{i}(x, y)=\sum_{\lambda \in \Lambda} q_{i}^{\lambda}(x, y)=\sum_{\lambda \in \Lambda} \hat{q}_{i}^{\lambda}(x) \varphi_{\lambda}(y)$. We define the (semi-)norms

$$
|Q|_{s}=\left(\sum_{\lambda \in \Lambda}\left|Q^{\lambda}\right|_{s}^{2}\right)^{1 / 2}
$$

where

$$
\left|Q^{\lambda}\right|_{s}=|\lambda|^{s}\left(\left\|q_{1}^{\lambda}\right\|_{L^{2}}^{2}+\left\|q_{2}^{\lambda}\right\|_{L^{2}}^{2}+\left\|q_{3}^{\lambda}\right\|_{L^{2}}^{2}\right)^{1 / 2}
$$

Frequently we write $|Q|_{0}=\|Q\|_{L^{2}},\left|Q^{\lambda}\right|_{0}=\left\|Q^{\lambda}\right\|_{L^{2}}$ and $\|Q\|_{k}=\left(\sum_{j=0}^{k}|Q|_{j}^{2}\right)^{1 / 2}$.

Theorem 5.1. Assume that $b \neq 0, \underline{u}^{0} \in\left[H_{p}^{5}(\Omega)\right]^{5}$. Then the asymptotic consistency error $e_{c, B}^{0}\left(\underline{u}_{0}\right)$, as defined by (3.10), satisfies

$$
e_{c, B}^{0}\left(\underline{u}_{0}\right) \leq C\left(\left\|\underline{u}_{0}\right\|_{5}+\|Q\|_{1}\right) h .
$$


Proof. Let $\underline{v} \in \mathcal{U}_{0, h}$ and write

$$
\begin{gathered}
\underline{v}=\sum_{\lambda \in \Lambda_{N}} \underline{\tilde{\vartheta}}_{\lambda}(x, y)=\sum_{\lambda \in \Lambda_{N}} A_{\lambda} \tilde{\varphi}_{\lambda}(y) \tilde{\tilde{\zeta}}_{\lambda}(x) \\
=\sum_{\lambda \in \Lambda_{N}} A_{\lambda} \tilde{\varphi}_{\lambda}(y)\left(\tilde{u}_{\lambda}(x), \tilde{v}_{\lambda}(x), \tilde{w}_{\lambda}(x), \tilde{\theta}_{\lambda}(x), \tilde{\psi}_{\lambda}(x)\right), \\
\quad \underline{u}_{0}=\sum_{\lambda \in \Lambda} \underline{u}_{0}^{\lambda}=\sum_{\lambda \in \Lambda}\left(u_{0}^{\lambda}, v_{0}^{\lambda}, w_{0}^{\lambda}, \theta_{0}^{\lambda}, \psi_{0}^{\lambda}\right)
\end{gathered}
$$

and $Q(\underline{v})=\sum_{\lambda \in \Lambda} Q^{\lambda}(\underline{v})$. Then by the orthogonality of the Fourier modes 3 ] we have that

$$
\begin{aligned}
\mathcal{A}_{b}\left(\underline{u}_{0}, \underline{v}\right)-Q(\underline{v})= & \mathcal{A}_{b}\left(\sum_{\lambda \in \Lambda} \underline{u}_{0}^{\lambda}, \sum_{\lambda \in \Lambda_{N}} \underline{\tilde{\vartheta}}_{\lambda}\right)-\sum_{\lambda \in \Lambda} Q^{\lambda}\left(\sum_{\lambda \in \Lambda_{N}} \underline{\tilde{\vartheta}}_{\lambda}\right) \\
= & \mathcal{A}_{b}\left(\sum_{|\lambda| \leq \lambda_{0}} \underline{u}_{0}^{\lambda}, \sum_{\lambda \in \Lambda_{N}} \underline{\tilde{\vartheta}}_{\lambda}\right)-\sum_{|\lambda| \leq \lambda_{0}} Q^{\lambda}\left(\sum_{\lambda \in \Lambda_{N}} \underline{\tilde{\vartheta}}_{\lambda}\right) \\
& +\mathcal{A}_{b}\left(\sum_{|\lambda|>\lambda_{0}} \underline{u}_{0}^{\lambda}, \sum_{\lambda \in \Lambda_{N}} \underline{\tilde{\vartheta}}_{\lambda}\right)-\sum_{|\lambda|>\lambda_{0}} Q^{\lambda}\left(\sum_{\lambda \in \Lambda_{N}} \underline{\tilde{\vartheta}}_{\lambda}\right) \\
= & \sum_{|\lambda| \leq \lambda_{0}}\left(\mathcal{A}_{b}\left(\underline{u}_{0}^{\lambda}, \underline{\tilde{\vartheta}}_{\lambda}\right)-Q^{\lambda}\left(\underline{\tilde{\vartheta}}_{\lambda}\right)\right)+\sum_{|\lambda|>\lambda_{0}}\left(\mathcal{A}_{b}\left(\underline{u}_{0}^{\lambda}, \underline{v}^{\prime}\right)-Q^{\lambda}(\underline{v})\right) \\
= & I+I I
\end{aligned}
$$

for any $\lambda_{0}$ such that $\lambda_{0} h_{y} \leq c<\pi$.

Let us first bound the term $I I$. Here we have

$$
\sum_{|\lambda|>\lambda_{0}} \mathcal{A}_{b}\left(\underline{u}_{0}^{\lambda}, \underline{v}\right) \leq \lambda_{0}^{-1} \sum_{|\lambda|>\lambda_{0}} \mathcal{A}_{b}\left(|\lambda| \underline{u}_{0}^{\lambda}, \underline{v}\right) \leq C h\left\|\underline{u}_{0}\right\|_{2}\left|\|\underline{v} \mid\|_{B, h}\right.
$$

for $\lambda_{0}=\frac{c}{h}, c$ sufficiently small, and similarly

$$
\sum_{|\lambda|>\lambda_{0}} Q^{\lambda}(\underline{v}) \leq \lambda_{0}^{-1} \sum_{|\lambda|>\lambda_{0}}|\lambda| Q^{\lambda}(\underline{v}) \leq C h|Q|_{1}\left|\|\underline{v} \mid\|_{B, h}\right.
$$

To bound the term $I$ when $b \neq 0$, we note that for any $\underline{\vartheta}_{\lambda}=A_{\lambda} \varphi_{\lambda} \underline{\zeta}_{\lambda} \in \mathcal{U}_{0}$ we can write

$$
\begin{aligned}
\mathcal{A}_{b}\left(\underline{u}_{0}^{\lambda}, \underline{\tilde{\vartheta}}_{\lambda}\right)-Q^{\lambda}\left(\underline{\tilde{\vartheta}}_{\lambda}\right) & =\mathcal{A}_{b}\left(\underline{u}_{0}^{\lambda}, \underline{\tilde{\vartheta}}_{\lambda}-\underline{\vartheta}_{\lambda}\right)-Q^{\lambda}\left(\underline{\tilde{\vartheta}}_{\lambda}-\underline{\vartheta}_{\lambda}\right) \\
& =A_{\lambda}\left(\mathcal{A}_{b}\left(\underline{u}_{0}^{\lambda}, \tilde{\varphi}_{\lambda} \underline{\tilde{\tilde{S}}}_{\lambda}-\varphi_{\lambda} \underline{\zeta}_{\lambda}\right)-Q^{\lambda}\left(\tilde{\tilde{\varphi}}_{\lambda} \underline{\tilde{\zeta}}_{\lambda}-\varphi_{\lambda} \underline{\zeta}_{\lambda}\right)\right) .
\end{aligned}
$$

Integration by parts in the first term in (5.3) gives

$$
\begin{aligned}
\mathcal{A}_{b}\left(\underline{u}_{0}^{\lambda}, \tilde{\varphi}_{\lambda} \underline{\tilde{\zeta}}-\varphi_{\lambda} \underline{\zeta}_{\lambda}\right)= & \left.\int_{0}^{H}\right|_{0} ^{L} \bar{\alpha}_{1}^{\lambda}\left(\tilde{\varphi}_{\lambda} \tilde{\theta}_{\lambda}-\varphi_{\lambda} \theta_{\lambda}\right)+\bar{\alpha}_{2}^{\lambda}\left(\tilde{\varphi}_{\lambda} \tilde{\psi}_{\lambda}-\varphi_{\lambda} \psi_{\lambda}\right) d y \\
& +\int_{\Omega} \bar{\delta}_{1}^{\lambda}\left(\tilde{\varphi}_{\lambda} \tilde{\theta}_{\lambda}-\varphi_{\lambda} \theta_{\lambda}\right)+\bar{\delta}_{2}^{\lambda}\left(\tilde{\varphi}_{\lambda} \tilde{\psi}_{\lambda}-\varphi_{\lambda} \psi_{\lambda}\right) d x d y
\end{aligned}
$$

where

$$
\left\{\begin{array}{l}
\alpha_{1}^{\lambda}=\frac{\partial^{2} w_{0}^{\lambda}}{\partial x^{2}}+\nu \frac{\partial^{2} w_{0}^{\lambda}}{\partial y^{2}}, \\
\alpha_{2}^{\lambda}=(1-\nu) \frac{\partial^{2} w_{0}^{\lambda}}{\partial x \partial y}, \\
\delta_{1}^{\lambda}=-\frac{\partial}{\partial x} \Delta w_{0}^{\lambda}, \\
\delta_{2}^{\lambda}=-\frac{\partial}{\partial y} \Delta w_{0}^{\lambda},
\end{array}\right.
$$


so that

$$
\begin{aligned}
& \mathcal{A}_{b}\left(\underline{u}_{0}^{\lambda}, \tilde{\varphi}_{\lambda} \underline{\tilde{\zeta}}-\varphi_{\lambda} \underline{\zeta}_{\lambda}\right) \leq\left\|\alpha_{1}^{\lambda}(L, \cdot)\right\|_{L^{2}(0, H)}\left\|\tilde{\varphi}_{\lambda} \tilde{\theta}_{\lambda}(L, \cdot)-\varphi_{\lambda} \theta_{\lambda}(L, \cdot)\right\|_{L^{2}(0, H)} \\
& \quad+\left\|\delta_{1}^{\lambda}(L, \cdot)\right\|_{L^{2}(0, H)}\left\|\tilde{\varphi}_{\lambda} \tilde{\psi}_{\lambda}(L, \cdot)-\varphi_{\lambda} \psi_{\lambda}(L, \cdot)\right\|_{L^{2}(0, H)} \\
& \quad+\left\|\alpha_{1}^{\lambda}(0, \cdot)\right\|_{L^{2}(0, H)}\left\|\tilde{\varphi}_{\lambda} \tilde{\theta}_{\lambda}(0, \cdot)-\varphi_{\lambda} \theta_{\lambda}(0, \cdot)\right\|_{L^{2}(0, H)} \\
& \quad+\left\|\delta_{1}^{\lambda}(0, \cdot)\right\|_{L^{2}(0, H)}\left\|\tilde{\varphi}_{\lambda} \tilde{\psi}_{\lambda}(0, \cdot)-\varphi_{\lambda} \psi_{\lambda}(0, \cdot)\right\|_{L^{2}(0, H)} \\
& \quad+\left\|\alpha_{2}^{\lambda}\right\|_{L^{2}}\left\|\tilde{\varphi}_{\lambda} \tilde{\theta}_{\lambda}-\varphi_{\lambda} \theta_{\lambda}\right\|_{L^{2}}+\left\|\delta_{2}^{\lambda}\right\|_{L^{2}}\left\|\tilde{\varphi}_{\lambda} \tilde{\psi}_{\lambda}-\varphi_{\lambda} \psi_{\lambda}\right\|_{L^{2}} \\
& \leq C\left\|\underline{u}_{0}^{\lambda}\right\|_{3}\left(\left\|\tilde{\varphi}_{\lambda} \tilde{\theta}_{\lambda}(L, \cdot)-\varphi_{\lambda} \theta_{\lambda}(L, \cdot)\right\|_{L^{2}(0, H)}\right. \\
&+\left\|\tilde{\varphi}_{\lambda} \tilde{\psi}_{\lambda}(L, \cdot)-\varphi_{\lambda} \psi_{\lambda}(L, \cdot)\right\|_{L^{2}(0, H)} \\
&+\left\|\tilde{\varphi}_{\lambda} \tilde{\theta}_{\lambda}(0, \cdot)-\varphi_{\lambda} \theta_{\lambda}(0, \cdot)\right\|_{L^{2}(0, H)} \\
&+\left\|\tilde{\varphi}_{\lambda} \tilde{\psi}_{\lambda}(0, \cdot)-\varphi_{\lambda} \psi_{\lambda}(0, \cdot)\right\|_{L^{2}(0, H)} \\
&\left.+\left\|\tilde{\varphi}_{\lambda} \tilde{\theta}_{\lambda}-\varphi_{\lambda} \theta_{\lambda}\right\|_{L^{2}}+\left\|\tilde{\varphi}_{\lambda} \tilde{\psi}_{\lambda}-\varphi_{\lambda} \psi_{\lambda}\right\|_{L^{2}}\right) .
\end{aligned}
$$

Also for $Q^{\lambda}$ in (5.3) we have the bound

$$
\begin{aligned}
Q^{\lambda}\left(\tilde{\varphi}_{\lambda} \underline{\tilde{\zeta}}_{\lambda}-\varphi_{\lambda} \underline{\zeta}_{\lambda}\right) \leq & C\left\|Q^{\lambda}\right\|_{L^{2}}\left(\left\|\tilde{\varphi}_{\lambda} \tilde{u}_{\lambda}-\varphi_{\lambda} u_{\lambda}\right\|_{L^{2}}\right. \\
& \left.+\left\|\tilde{\varphi}_{\lambda} \tilde{v}_{\lambda}-\varphi_{\lambda} v_{\lambda}\right\|_{L^{2}}+\left\|\tilde{\varphi}_{\lambda} \tilde{w}_{\lambda}-\varphi_{\lambda} w_{\lambda}\right\|_{L}^{2}\right) .
\end{aligned}
$$

To continue we need the following approximation results. The proof will be postponed to the end of this section.

Lemma 5.2. For every $\lambda$ such that $|\lambda| h_{y} \leq c<\pi$ there exists a $\varphi_{\lambda} \underline{\zeta}_{\lambda} \in \mathcal{U}_{0}$ such that

$$
\begin{aligned}
\left\|\tilde{\varphi}_{\lambda} \tilde{\theta}_{\lambda}(L, \cdot)-\varphi_{\lambda} \theta_{\lambda}(L, \cdot)\right\|_{L^{2}(0, H)}+\left\|\tilde{\varphi}_{\lambda} \tilde{\psi}_{\lambda}(L, \cdot)-\varphi_{\lambda} \psi_{\lambda}(L, \cdot)\right\|_{L^{2}(0, H)} \\
\quad+\left\|\tilde{\varphi}_{\lambda} \tilde{\theta}_{\lambda}(0, \cdot)-\varphi_{\lambda} \theta_{\lambda}(0, \cdot)\right\|_{L^{2}(0, H)}+\left\|\tilde{\varphi}_{\lambda} \tilde{\psi}_{\lambda}(0, \cdot)-\varphi_{\lambda} \psi_{\lambda}(0, \cdot)\right\|_{L^{2}(0, H)} \\
\quad+\left\|\tilde{\varphi}_{\lambda} \tilde{\theta}_{\lambda}-\varphi_{\lambda} \theta_{\lambda}\right\|_{L^{2}}+\left\|\tilde{\varphi}_{\lambda} \tilde{\psi}_{\lambda}-\varphi_{\lambda} \psi_{\lambda}\right\|_{L^{2}} \\
\leq C\left(h^{2}|\lambda|^{5-m}+h^{2} \lambda^{4}\right)+C h \lambda^{2}\left\|\mid \tilde{\varphi}_{\lambda} \tilde{\tilde{\zeta}}_{\lambda}\right\| \|_{B, h}
\end{aligned}
$$

and

$$
\left\|\tilde{\varphi}_{\lambda} \tilde{u}_{\lambda}-\varphi_{\lambda} u_{\lambda}\right\|_{L^{2}}+\left\|\tilde{\varphi}_{\lambda} \tilde{v}_{\lambda}-\varphi_{\lambda} v_{\lambda}\right\|_{L^{2}}+\left\|\tilde{\varphi}_{\lambda} \tilde{w}_{\lambda}-\varphi_{\lambda} w_{\lambda}\right\|_{L^{2}} \leq C h^{2}|\lambda|^{4-3 m / 2}
$$

where $m=1$ in the elliptic case and $m=0$ in the hyperbolic and parabolic cases.

To complete the proof of Theorem[5.1 we note that by virtue of the inequalities $|\lambda|^{3-m / 2} \leq C\left|\left\|\tilde{\varphi}_{\lambda} \tilde{\zeta}_{\lambda} \mid\right\|_{B, h}\right.$ and $| \lambda \mid h \leq \lambda_{0} h \leq c<\pi$ we obtain from (5.4) with the help of Lemma 5.2

$$
\mathcal{A}_{b}\left(\underline{u}_{0}^{\lambda}, \tilde{\varphi}_{\lambda} \underline{\tilde{\zeta}}-\varphi_{\lambda} \underline{\zeta}_{\lambda}\right) \leq C h|| \lambda^{2} \underline{u}_{0}^{\lambda}\left\|_{3}\right\|\left|\tilde{\varphi}_{\lambda} \underline{\tilde{\zeta}}_{\lambda}\right| \|_{B, h}
$$

and from (5.5)

$$
Q^{\lambda}\left(\tilde{\varphi}_{\lambda} \underline{\tilde{\zeta}}_{\lambda}-\varphi_{\lambda} \underline{\zeta}_{\lambda}\right) \leq C h\left\|Q^{\lambda}\right\|_{L^{2}}\left\|\left|\tilde{\varphi}_{\lambda} \underline{\tilde{\zeta}}_{\lambda}\right|\right\| \|_{B, h}
$$


so that by (5.3), (5.8) and (5.9)

$$
\begin{aligned}
& \sum_{|\lambda| \leq \lambda_{0}}\left(\mathcal{A}_{b}\left(\underline{u}_{0}^{\lambda}, \underline{\tilde{\vartheta}}_{\lambda}\right)-Q^{\lambda}\left(\underline{\tilde{\vartheta}}_{\lambda}\right)\right)=\sum_{|\lambda| \leq \lambda_{0}} A_{\lambda}\left(\mathcal{A}_{b}\left(\underline{u}_{0}^{\lambda}, \tilde{\varphi}_{\lambda} \underline{\tilde{\zeta}}_{\lambda}\right)-Q^{\lambda}\left(\tilde{\varphi}_{\lambda} \underline{\tilde{S}}_{\lambda}\right)\right) \\
& \quad \leq C h \sum_{|\lambda| \leq \lambda_{0}}\left(\left\|\lambda^{2} \underline{u}_{0}^{\lambda}\right\|_{3}+\left\|Q^{\lambda}\right\|_{L^{2}}\right)\left\|A_{\lambda} \tilde{\varphi}_{\lambda} \underline{\tilde{\zeta}}_{\lambda} \mid\right\|_{B, h} \\
& \quad \leq C h\left(\left\|\underline{u}_{0}\right\|_{5}+\|Q\|_{L^{2}}\right)\left|\left\|\underline{v}|\||_{B, h},\right.\right.
\end{aligned}
$$

and Theorem 5.1 follows from the estimates (5.1), (5.2) and (5.10).

Proof of Lemma 5.2. In [3] it was shown that for every discrete mode $\tilde{\varphi}_{\lambda} \underline{\tilde{\zeta}}_{\lambda} \in \mathcal{U}_{0, h}$ with $|\lambda| h_{y} \leq c<\pi$ there corresponds a continuous mode

$$
\varphi_{\lambda} \underline{\zeta}_{\lambda}=\varphi_{\lambda}(y)\left(u_{\lambda}(x), v_{\lambda}(x), w_{\lambda}(x), \theta_{\lambda}(x), \psi_{\lambda}(x)\right) \in \mathcal{U}_{0}
$$

satisfying $u_{\lambda}(0)=\tilde{u}_{\lambda}(0)$ and $v_{\lambda}(0)=\tilde{v}_{\lambda}(0)$ and such that

$$
\begin{aligned}
&\left|u_{\lambda}\left(x^{k}\right)-\tilde{u}_{\lambda}\left(x^{k}\right)\right| \leq C h^{2}|\lambda|^{3-m} e^{-\beta|\lambda| x^{k}}, \\
&\left|v_{\lambda}\left(x^{k}\right)-\tilde{v}_{\lambda}\left(x^{k}\right)\right| \leq C h^{2}|\lambda|^{3-m} e^{-\beta|\lambda| x^{k}}, \\
&\left|w_{\lambda}\left(x^{k}\right)-\tilde{w}_{\lambda}\left(x^{k}\right)\right| \leq C h^{2}|\lambda|^{4-m} e^{-\beta|\lambda| x^{k}}, \\
&\left|\psi_{\lambda}\left(x^{k}\right)-\tilde{\psi}_{\lambda}\left(x^{k}\right)\right| \leq C h^{2}|\lambda|^{5-m} e^{-\beta|\lambda| x^{k}},
\end{aligned}
$$

with $\beta>0$ in the elliptic case and $\beta=0$ in the hyperbolic and parabolic cases, so that

$$
\begin{aligned}
\left\|\tilde{\varphi}_{\lambda} \tilde{\psi}_{\lambda}(0, \cdot)-\varphi_{\lambda} \psi_{\lambda}(0, \cdot)\right\|_{L^{2}(0, H)} & \leq C h^{2}|\lambda|^{5-m}, \\
\left\|\tilde{\varphi}_{\lambda} \tilde{\psi}_{\lambda}(L, \cdot)-\varphi_{\lambda} \psi_{\lambda}(L, \cdot)\right\|_{L^{2}(0, H)} & \leq C h^{2}|\lambda|^{5-m}, \\
\left\|\tilde{\varphi}_{\lambda} \tilde{\psi}_{\lambda}-\varphi_{\lambda} \psi_{\lambda}\right\|_{L^{2}} & \leq C h^{2}|\lambda|^{5-3 m / 2},
\end{aligned}
$$

and

$$
\begin{aligned}
&\left\|\tilde{\varphi}_{\lambda} \tilde{u}_{\lambda}-\varphi_{\lambda} u_{\lambda}\right\|_{L^{2}} \leq C h^{2}|\lambda|^{3-3 m / 2}, \\
&\left\|\tilde{\varphi}_{\lambda} \tilde{v}_{\lambda}-\varphi_{\lambda} v_{\lambda}\right\|_{L^{2}} \leq C h^{2}|\lambda|^{3-3 m / 2}, \\
&\left\|\tilde{\varphi}_{\lambda} \tilde{w}_{\lambda}-\varphi_{\lambda} w_{\lambda}\right\|_{L^{2}} \leq C h^{2}|\lambda|^{4-3 m / 2} .
\end{aligned}
$$

Also, by 3$]$ we have that

$$
\begin{aligned}
\frac{1}{2}\left(\tilde{\theta}_{\lambda}\left(x^{k+1}\right)+\tilde{\theta}_{\lambda}\left(x^{k}\right)\right)= & \frac{2}{b h_{y}^{2}} \tan ^{2}\left(\frac{1}{2} \lambda h_{y}\right)\left(\frac{2 c}{b}\left(\tilde{v}_{\lambda}\left(x^{k+1}\right)+\tilde{v}_{\lambda}\left(x^{k}\right)\right)\right. \\
& \left.-\left(\tilde{u}_{\lambda}\left(x^{k+1}\right)+\tilde{u}_{\lambda}\left(x^{k}\right)\right)\right) \\
= & \frac{1}{2}\left(\tilde{g}\left(x^{k+1}\right)+\tilde{g}\left(x^{k}\right)\right)
\end{aligned}
$$

so that

$$
\tilde{\theta}_{\lambda}\left(x^{k+1}\right)=\tilde{g}\left(x^{k+1}\right)+(-1)^{k}\left(\tilde{\theta}_{\lambda}\left(x^{0}\right)-\tilde{g}\left(x^{0}\right)\right)
$$


and

$$
\begin{aligned}
\frac{\partial \tilde{\theta}_{\lambda}}{\partial x}\left(x^{k+1 / 2}\right) & =\frac{\tilde{\theta}_{\lambda}\left(x^{k+1}\right)-\tilde{\theta}_{\lambda}\left(x^{k}\right)}{h_{x}^{k}} \\
& =\frac{\tilde{g}\left(x^{k+1}\right)-\tilde{g}\left(x^{k}\right)}{h_{x}^{k}}+\frac{2}{h_{x}^{k}}(-1)^{k}\left(\tilde{\theta}_{\lambda}\left(x^{0}\right)-\tilde{g}\left(x^{0}\right)\right) .
\end{aligned}
$$

Since

$$
\theta_{\lambda}\left(x^{k}\right)=\frac{\lambda^{2}}{b}\left(\frac{2 c}{b} v_{\lambda}\left(x^{k}\right)-u_{\lambda}\left(x^{k}\right)\right)=g\left(x^{k}\right),
$$

it follows from $(5.13)-(5.15)$ that

$$
\begin{aligned}
\tilde{\theta}_{\lambda}\left(x^{k+1}\right)-\theta_{\lambda}\left(x^{k+1}\right)= & \tilde{g}\left(x^{k+1}\right)-g\left(x^{k+1}\right) \\
& +\frac{h_{x}^{k}}{2} \frac{\partial \theta_{\lambda}}{\partial x}\left(x^{k+1 / 2}\right)-\frac{h_{x}^{k}}{2} \frac{\tilde{g}\left(x^{k+1}\right)-\tilde{g}\left(x^{k}\right)}{h_{x}^{k}},
\end{aligned}
$$

and finally that

$$
\begin{aligned}
\left|\tilde{\theta}_{\lambda}\left(x^{k+1}\right)-\theta_{\lambda}\left(x^{k+1}\right)\right| \leq & C\left(h^{2}|\lambda|^{5-m} e^{-\beta|\lambda| x^{k+1}}+h\left|\frac{\partial \tilde{\theta}_{\lambda}}{\partial x}\left(x^{k+1 / 2}\right)\right|\right. \\
& +h \lambda^{2}\left(\left|\frac{\partial \tilde{v}_{\lambda}}{\partial x}\left(x^{k+1 / 2}\right)\right|+\left|\frac{\partial \tilde{u}_{\lambda}}{\partial x}\left(x^{k+1 / 2}\right)\right|\right) .
\end{aligned}
$$

For the values at the end-points we get similarly

$$
\begin{aligned}
\tilde{\theta}_{\lambda}\left(x^{0}\right)-\theta_{\lambda}\left(x^{0}\right)= & (-1)^{k}\left(\tilde{\theta}_{\lambda}\left(x^{k+1}\right)-\theta_{\lambda}\left(x^{k+1}\right)+g\left(x^{k+1}\right)-\tilde{g}\left(x^{k+1}\right)\right) \\
& +\tilde{g}\left(x^{0}\right)-g\left(x^{0}\right)
\end{aligned}
$$

and

$$
\begin{aligned}
\tilde{\theta}_{\lambda}\left(x^{N_{x}}\right)-\theta_{\lambda}\left(x^{N_{x}}\right)= & (-1)^{N_{x}}\left(\tilde{\theta}_{\lambda}\left(x^{0}\right)-\theta_{\lambda}\left(x^{0}\right)+g\left(x^{0}\right)-\tilde{g}\left(x^{0}\right)\right) \\
& +\tilde{g}\left(x^{N_{x}}\right)-g\left(x^{N_{x}}\right) .
\end{aligned}
$$

Thus, we have the following bounds:

$$
\begin{aligned}
\left\|\tilde{\varphi}_{\lambda} \tilde{\theta}_{\lambda}(0, \cdot)-\varphi_{\lambda} \theta_{\lambda}(0, \cdot)\right\|_{L^{2}(0, H)} \leq & C\left(h^{2} \lambda^{4}+h^{2}|\lambda|^{5-3 m / 2}\right) \\
& +C\left(h+h \lambda^{2}\right)\left\|\left|\tilde{\varphi}_{\lambda} \underline{\tilde{\zeta}}_{\lambda}\right|\right\|_{B, h}, \\
\left\|\tilde{\varphi}_{\lambda} \tilde{\theta}_{\lambda}(L, \cdot)-\varphi_{\lambda} \theta_{\lambda}(L, \cdot)\right\|_{L^{2}(0, H)} \leq & C\left(h^{2} \lambda^{4}+h^{2}|\lambda|^{5-3 m / 2}\right) \\
& +C\left(h+h \lambda^{2}\right)\left\|\tilde{\varphi}_{\lambda} \tilde{\tilde{S}}_{\lambda} \mid\right\|_{B, h} \\
& +C\left(h^{2}|\lambda|^{5-m}+h^{2} \lambda^{4}\right), \\
\left\|\tilde{\varphi}_{\lambda} \tilde{\theta}_{\lambda}-\varphi_{\lambda} \theta_{\lambda}\right\|_{L^{2}} \leq & C h^{2}|\lambda|^{5-3 m / 2} \\
& +C\left(h+h \lambda^{2}\right)\left\|\left|\tilde{\varphi}_{\lambda} \underline{\tilde{\zeta}}_{\lambda}\right|\right\|_{B, h} .
\end{aligned}
$$

Lemma 5.2 follows from (5.11), (5.12) and (5.16), since $|\lambda| h_{y} \leq c<\pi$. 


\section{Conclusions}

The general conclusion from Parts I and II of the paper is that, at least under extremely favorable circumstances, both bending- and membrane-dominated smooth deformations can be approximated with nearly optimal accuracy by the bilinear MITC4 element. To be more precise, under the conditions presented above and in [3] the approximation errors are bounded by

$$
\begin{aligned}
& e_{a, M} \leq C h\|\underline{u}\|_{2}, \\
& e_{a, B} \leq C_{1} h\|\underline{u}\|_{6}+C_{2} h^{s-2} t^{-1}\|\underline{u}\|_{s}, \quad s \geq 6,
\end{aligned}
$$

and if $\underline{u}$ is an inextensional deformation the bound

$$
\tilde{e}_{a}=\min _{\underline{v} \in \mathcal{U}_{0, h}}|||\underline{u}-\underline{v}|||_{B, h} \leq C_{1} h|\underline{u}|_{1}+C_{2} h^{\frac{2}{3}(s-1)}|\underline{u}|_{s}, \quad 2 \leq s \leq 3
$$

holds. Here $C_{2}=0$ in the elliptic case and in the degenerate parabolic and hyperbolic cases.

For the consistency errors, only the case when the geometric parameter $b$ is nonzero is considered. In this case, again under very restrictive conditions, the results are as in Theorem 4.4. Finally, the asymptotic consistency error is bounded in Theorem 5.1 .

From the results quoted above, one can conclude that the MITC-type elements manage well in approximating the inextensional deformations and thus in relieving the locking effects. This is probably just the task they were designed for. On the other hand, the modifications bring along consistency error components, and it is necessary to impose several assumptions on the smoothness of the solution to obtain reasonable convergence rates. Judging from these results, it appears as if the design of the element was oriented more towards the bending-dominated deformation states than membrane-dominated ones. However, it should be remembered that attempts to design a general low-order shell element have so far inevitably lead to compromises.

Open questions still remain. First of all, to what extent do the results obtained so far hold for more general geometries, deformation states, and finite element meshes? Second, the ability of the MITC4 element to capture the boundary layers, virtually always present in thin shells, is unknown.

\section{REFERENCES}

1. K.J. Bathe, E.N. Dvorkin, A formulation of general shell elements - the use of mixed interpolation of tensorial components, Int. J. Numer. Methods Engrg. 22 (1986) 697-722.

2. S.C. Brenner, L.R. Scott, The Mathematical Theory of Finite Element Methods (Springer, 1994) MR 95f:65001

3. V. Havu, J. Pitkäranta, Analysis of a bilinear finite element for shallow shells I: Approximation of inextensional deformations, Math. Comp. 71 (2002) 923-943.

4. M. Malinen, On the classical shell model underlying bilinear degenerated shell finite elements, Int. J. Numer. Methods Engrg. 52 (2001) 389-416.

5. J. Pitkäranta, Y. Leino, O. Ovaskainen, J. Piila, Shell deformation states and the finite element method: a benchmark study of cylindrical shells Comput. Methods Appl. Mech. Engrg. 128 (1995) 81-121 MR 96j:73085 
6. J. Pitkäranta, The first locking-free plane-elastic finite element: historia mathematica, Helsinki University of Technology Institute of Mathematics Research Reports A411 (1999). Cf. MR 2001m:74064

7. J. Pitkäranta, The problem of membrane locking in finite element analysis of cylindrical shells, Numer. Math. 61, (1992) 523-542. MR 93b:65178

Institute of Mathematics, Helsinki University of Technology, P.O. Box 1100, 02015 Helsinki University of Technology, Finland

E-mail address: Ville.Havu@hut.fi

Institute of Mathematics, Helsinki University of Technology, P.O. Box 1100, 02015 Helsinki University of Technology, Finland

E-mail address: Juhani.Pitkaranta@hut.fi 